\title{
Using the anatomical tibial axis for total knee arthroplasty alignment may lead to an internal rotation error
}

\author{
Csaba Forster-Horvath ${ }^{1}$ - Valerie Kremo ${ }^{2}$ - Magdalena Müller-Gerbl ${ }^{2}$. \\ Andrej Maria Nowakowski ${ }^{1,2}$
}

Received: 15 April 2015 / Accepted: 15 April 2015 /Published online: 9 July 2015

(C) SICOT aisbl 2015

\begin{abstract}
Introduction Despite intensive research, current total knee arthroplasty (TKA) designs do not always provide the correct kinematics for the native joint and thus further optimisation is necessary. Several studies support the importance of malrotation of the tibial components in the failure of TKA. We hypothesise that using the anatomical tibial axis (ATA) to align tibial component rotation on the resected tibial surface may lead to an internal rotation error due to relative anterior shift of the lateral articular surface centre compared to the medial one. The aim of this study was to compare the anatomical tibial axis of the physiological tibial joint surface to the resected one.

Method Twenty formalin-fixed cadaveric knees were obtained for study. After computed tomography scanning the data of each specimen were entered into a standardised coordinate system and virtual bone cuts were performed with 6,8 and $10 \mathrm{~mm}$ resection depths. The positions of the articular surface centres were determined at each resection depth.

Results The lateral articular surface centre had moved anteriorly after the resection by a mean $1.475 \mathrm{~mm}$, while the medial one had not changed significantly. Resecting the tibia at a 6$\mathrm{mm}$ cut and using the transverse tibial axis to align the prosthetic tibial plateau will result in a mean $4.0^{\circ}(95 \%$ confidence
\end{abstract}

Csaba Forster-Horvath and Valerie Kremo contributed equally to this work

Andrej Maria Nowakowski

andrej.nowakowski@unibas.ch

1 Orthopaedic Department, University of Basel, Spitalstrasse 21, 4031 Basel, Switzerland

2 Institute of Anatomy, University of Basel, Pestalozzistrasse 20, 4056 Basel, Switzerland interval, $2.5-5.5^{\circ}$ ) of internal rotation compared to the uncut tibia.

Discussion The ATA lies in 6 degrees of external rotation compared to the perpendicular to the posterior tibial condylar axis (PTCA). Graw et al. suggest aligning the tibial component in 10 degrees of external rotation to the latter. Thus, if we accept the above suggestion, the ATA is 4 degrees internally rotated compared to the same line on the resected proximal tibia. These prior studies appear to be in accordance with our findings.

Conclusions We conclude that using the ATA on the resected tibial surface may contribute to an internal rotation error.

Keywords Total knee replacement · Total knee arthroplasty · Anatomicalal tibial axis · Transverse tibial axis · Tibial component alignment · Tibial component malrotation

$\begin{array}{ll}\text { Abbreviations } \\ \text { ATA } & \text { Anatomical tibial axis } \\ \text { FFC } & \text { Flexion facet centre } \\ \text { PTCA } & \text { Posterior tibial condylar axis } \\ \text { TKA } & \text { Total knee arthroplasty } \\ \text { TRP } & \text { Tibial reference points }\end{array}$

\section{Introduction}

Physical function, pain and vitality improve significantly after total knee arthroplasty (TKA); however, unlike total hip replacements, these symptoms in the mid term often remain worse compared with age-matched healthy controls $[1,2]$.

In the case of TKA, a significant proportion of patients are dissatisfied with the outcome of surgery [3]. These findings are supported by specific patient reported outcome measures 
designed for patients with knee pathologies [4]. One of the possible explanations lies within the complexity of the kinematics of the knee joint. Despite intensive research, current TKA designs do not provide the kinematics of the native joint and thus further optimisation is needed, such as the improvement of the alignment of implant components [5, 6].

Between 1990 and 2002, 8.2\% of all knee arthroplasty operations in the USA were revision procedures [7]. The proportion of revision surgery due to malalignment lies between $2.9 \%[8]$ and $11.8 \%$ [9].

Malalignment is one of the most frequent reasons for revision, ranking fifth after aseptic loosening, pain, infection and instability also according to the 11th annual report of 2014 of the National Joint Registry of England and Wales.

Of note is that malalignment is responsible for implant wear, instability and unexplained pain in some cases, which may result in under-reporting of this category. Other studies support the importance of malrotation of the tibial components in the failure of TKA $[8,10,11]$.

Stiff knees after TKA have been assessed for malrotation of any of the components and tibial component internal rotation was found to be significantly more frequently associated with this condition [12].

Also the patellofemoral kinematics may be severely disturbed by internal malrotation of the tibial component [13].

Internal rotational malpositioning is far more frequent and shows a significantly bigger range compared with an external rotation error [12, 14].

Both femoral and tibial component malposition are responsible for malalignment and often a combined error can be found. With the measured resection technique, anatomical landmarks guide the surgeon planning the bone cuts [15]. The difficulty of this technique is to precisely identify these landmarks $[16,17]$.

Malalignment of the femoral component may result in flexion gap disbalance and-more importantly—patellofemoral maltracking.

When a measured resection technique is used, the surgeon relies on several anatomical landmarks when referencing the rotation of the femoral component.

The sulcus line of the trochlear groove, the Whiteside line [18], the surgical epicondylar axis [19] and the posterior condylar axis with a higher variability are used.

Also, in the case of the tibial component rotation, several anatomical landmarks have been proposed and yet none of them have been recognised as the ultimate reference [10, 20]. The medial edge of the tibial tuberosity [21-25] has been shown to be less reliable than the medial third of the tibial tuberosity [22, 23, 26]. Siston et al. [23] found that using the medial edge and the posterior cruciate attachment as reference causes internal rotation of the tibial component.

The posterior tibial condylar line [20,24, 26, 27], the transverse axis of the tibia, the patellar tendon [20, 27-29], the malleolar axis [20, 22, 24, 27, 29], sulcus of the intercondylar eminences [22] and the second metatarsal [23] have all been described as anatomical landmarks for correct tibial component rotation.

The important question is: which factors contribute to tibial component malrotation?

The rotational alignment of the tibial component is determined on the resected tibial surface by eyeballing the surface landmarks and the contour of the medial and lateral tibial condyles.

If the surgeon is following the principle of best fit and coverage of the resected bone surface, then he or she would place the tibial component symmetrically between the anterior and posterior condylar margins on the medial tibial plateau. In this study, we deliver an explanation for an internal rotation error introduced by this technique.

We postulate that using the anatomical tibial axis (ATA) to align tibial component rotation on the resected tibial surface may lead to an internal rotation error due to the anterior shift of the lateral articular surface centre compared to the medial one at the level of resection.

\section{Materials and methods}

To prove this hypothesis, we determined the medial and lateral articular surface centres of the resected tibia and compared them with the unresected proximal articular surfaces using a computed tomography (CT)-based coordinate system.

The research adhered to the tenets of the Declaration of Helsinki. After approval by the Ethics Commission Beider Basel (EKBB) of Switzerland 20 (11 left and 9 right with a mixture of male and female) formalin-fixed knee joint specimens from a university-level anatomy course were investigated. The mean age of the donors was 84.8 years ( \pm 10.8 years), the mean body weight was $63.4 \mathrm{~kg}( \pm 15.2 \mathrm{~kg})$ and the mean body height was $162.6 \mathrm{~cm}( \pm 11.2 \mathrm{~cm})$. Specimens were from four male and eight female donors. Tibiae with significant osteoarthritis were excluded.

Imaging was performed with a helical GE Lightspeed 16 row CT scanner (General Electric Healthcare Corporation, Waukesha, WI, USA): $120 \mathrm{kV}$, slice thickness $0.625 \mathrm{~mm}$, voxel depth $0.5 \mathrm{~mm}$, voxel height $0.283203 \mathrm{~mm}$ and voxel width $0.283203 \mathrm{~mm}$.

Adjustment of data using a standardised coordinate system was as described by Kaech and Müller-Gerbl [30].

The DICOM (Digital Imaging and Communications in Medicine, Rosslyn, VA, USA) data were analysed using the visualisation software VGStudio Max 2.1.1 (Volume Graphics, Heidelberg, Germany), which allows highprecision measurements by means of a CT-based coordinate measurement technology [31]. 
The data from the knee specimens were imported into a standardised coordinate system, which was based on the reports of Grood et al. [32] and McPherson et al. [33].

Two-dimensional reconstructions of the data sets in the sagittal, frontal, and transverse planes as well as a threedimensional reconstruction of the entire data volume per knee and axis were selected for monitor display.

The transverse flexion-axis was determined by measuring movements of the flexion facet centre (FFC) on the posterior femoral condyle. In the sagittal plane, tibial reference points (TRP) were determined [22]. The TRP is the intersection between the three spatial axes at the most distal edge of the posterior tibia. The FFC and TRP span the frontal plane. The coordinate system could be established from the frontal plane (primary reference), the axis through the FFC (secondary reference) and the TRP as the origin (tertiary reference).

After setting up the co-ordinate system, the tibia was isolated by defining it as region of interest (ROI) in order to achieve a free perspective on the uncut proximal tibial joint surface.

\section{Virtual bone resection and measurement}

Medial and lateral articular surface centres of the uncut and resected tibia were determined by calculating the root-meansquare of the error for the best-fit circle [34] (Figs. 1 and 2).

Virtual bone resections were performed according to the descriptions of Cheng et al. [35].

Resection of the tibia was carried out 6,8 and $10 \mathrm{~mm}$ distal to the deepest point of the medial tibial plateau parallel to the "horizontal plane" (Fig. 3).

The 6-mm cut corresponds to the level of the surgical tibial resection in an arthritic varus malaligned knee. This tibial cut was defined as the $0^{\circ}$ slope plane according to the standardised coordinate system.



Fig. 1 Physiological proximal tibial joint surface with articular surface centres

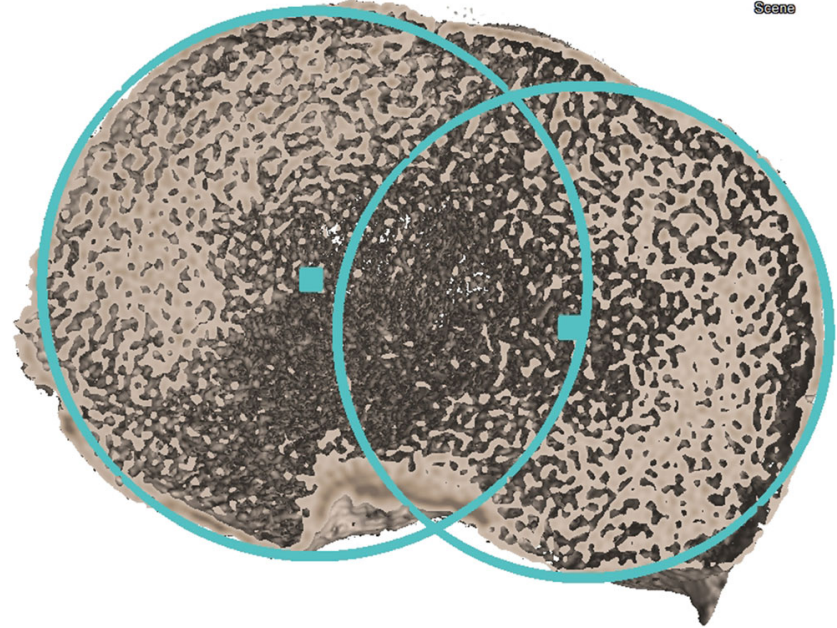

Fig. 2 Resected proximal tibia surface at $6 \mathrm{~mm}$ below the deepest point of the medial articular surface centre of a non-arthritic knee

In order to see how much internal rotation this will resulted in, we calculated the difference of coordinates in the sagittal plane by subtraction of the $6 \mathrm{~mm}$ cut data from the physiological ones $(y)$. Using the distance of the articular surface centres in the frontal plane of the 6-mm cut $(x)$, we then calculated the inverse tangent of $y / x$ to get the degree of internal rotation in each of the 20 cases. The mean, standard deviation and $95 \%$ confidence interval $(\mathrm{CI})$ were calculated.

\section{Statistical methods}

Data of three different section planes $(6,8$ and $10 \mathrm{~mm})$ plus the physiological tibia surface were taken for each knee.

In each case, circles with the above-mentioned method [34] were fitted to the medial and lateral tibial condyle and the radius and coordinates were assessed. The mean and standard deviation of these data were calculated. The results of the cut surfaces in three planes were compared to those of the uncut

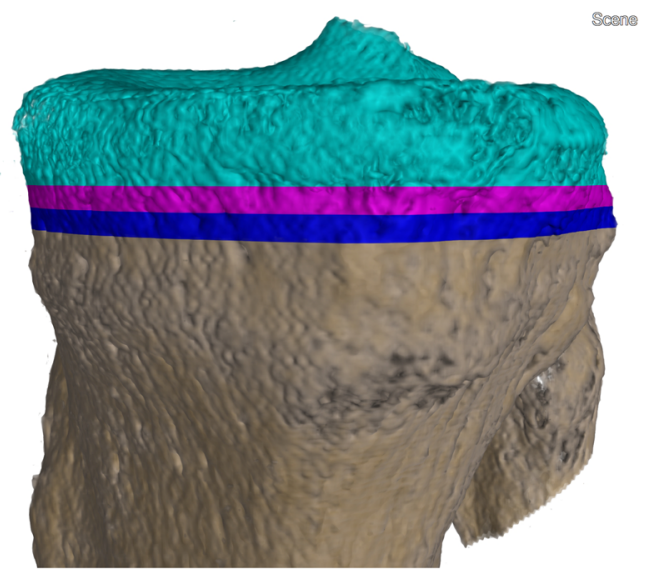

Fig. 3 Resection depths at 6,8 and $10 \mathrm{~mm}$ below the deepest point of the medial articular surface centre 
Fig. 4 Position of the articular surface centres in the sagittal plane ( $y$-coordinates in millimetres) depending on the height of tibial resection. Positive anterior $y$-axis direction
Sagittal position of articular surface centers depending on resection depth



tibia and the calculation was made using JMP Software (SAS Institute, Cary, NC, USA). The datasets were checked for normal distribution. Since our data were not normally distributed, Wilcoxon signed-rank test has been applied to show significance. Statistical significance was set at a $P$ value $\leq$ 0.05 .

\section{Results}

The mean lateral articular surface centre of the resected tibia was significantly more anterior than that of the physiological tibial surface $(P<0.0001)$ (Figs. 4 and 5). The lateral articular surface centre moved anteriorly by the resection, while the medial one did not change significantly, resulting in a relative shift of a mean $1.475 \mathrm{~mm}$.

The biggest change has been found between the physiological and the 6-mm cut. Further cuts at 8 and $10 \mathrm{~mm}$ show further anterior shift of the lateral articular surface centre; however this was not significant.

We calculated the extent of internal rotation error introduced by the relative shift of the articular surface centres. Using the $6 \mathrm{~mm}$ cut and using the transverse tibial axis to align the prosthetic tibial plateau will result in a mean $4.0^{\circ}(95 \% \mathrm{CI}$, $2.5-5.5^{\circ}$ ) of internal rotation compared with the uncut tibia.

\section{Discussion}

Patients with anterior knee pain compared with those with painless TKAs have $6.2^{\circ}$ versus $0.4^{\circ}$ of tibial internal rotation [13].

Since posterior motion of the femoral condyle on the tibial articular surface dominantly occurs on the lateral side, internal rotation of the tibial component does change tibio-femoral kinematics by limiting rollback, especially with more conforming inlay designs, thus potentially limiting flexion $[12,36]$.

These facts raise the question as to which landmarks to use in order to properly position the tibial component.

Using the contours of the medial and lateral tibial condyles, the centres of each condyle can be determined. Connecting these points represents the anatomical tibial axis [34], which may well influence the intra-operative decision in finalising the rotation of the tibial component.

If the surgeon uses a symmetrical tibial component and attempts to avoid posterolateral overhang and impingement of the popliteal tendon, the size of the tibial component should be chosen to fit the lateral condyle in the sagittal plane.

At the same time, the larger AP diameter of the medial condyle allows more freedom to set the final position of the component, and if the surgeon chooses to place it symmetrically between the anterior and posterior condylar margin,
Fig. 5 Relative anterior shift of the position ( $y$-coordinates in millimetres) of the articular surface centres in the sagittal plane compared to the physiological joint surface depending on the level of resection. Positive anterior $y$-axis direction. Significant shift of the lateral versus medial articular surface centres $(P<0.0001)$
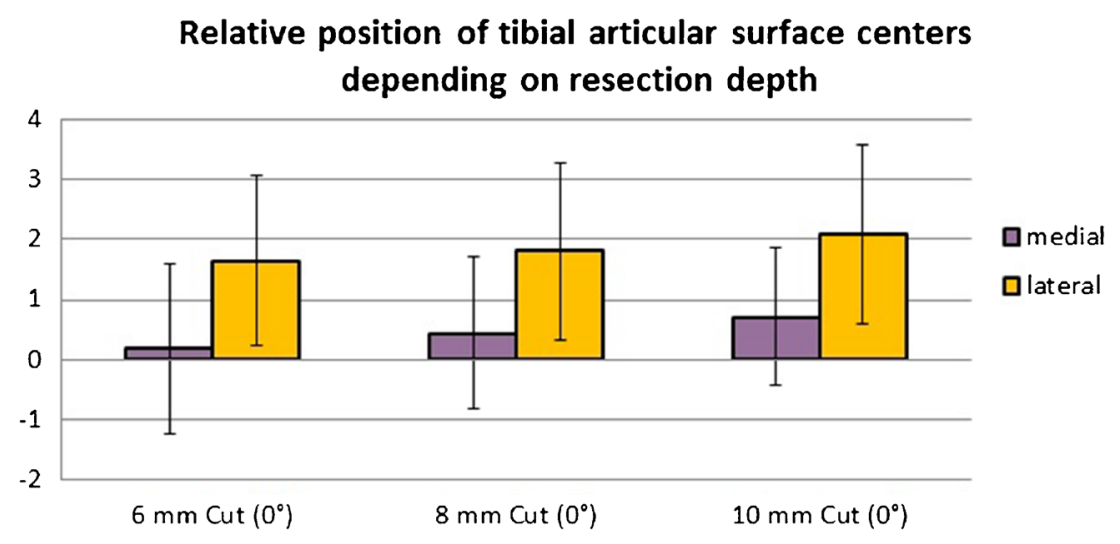
matching the medial articular surface centre, then the prosthetic tibial plateau will be placed in an internally rotated position in the horizontal plane.

In our study, we postulated that the line connecting the articular surface centres of the resected tibia is more internally rotated than that of the unresected tibia.

We found a significant anterior shift of the lateral articular surface centre versus the medial one at a resection depth applied in TKA.

The lateral articular surface centre moves anteriorly by the resection, while the medial one does not change significantly, resulting in a relative shift of a mean $1.5 \mathrm{~mm}$.

We found the internal rotation error introduced by the articular surface centres of the resected tibia at a mean $4.0^{\circ}$ (95\% CI, 2.5-5.5 ) compared with the uncut tibia.

Cobb et al. [34] described a method to reliably determine the rotation of the proximal tibia based on the centres of the unresected lateral and the virtually resected medial tibial plateaus - in order to gain a circular cortical contour-20 mm below tibial spine level.

Cobb et al. named the axis connecting these points the anatomical tibial axis (ATA), which shows a mean intra- and inter-observer variability of, respectively, $1.44^{\circ}$ and $1.66^{\circ}$.

From a previous study, we know that the medial condylar centre of the normal tibia lies significantly more anterior than its lateral counterpart, supporting the studies of Cobb et al. [34].

Since we found that the medial articular surface centre, unlike the lateral one, does not significantly shift anteriorly with resection, we may conclude that the ATA on the resected tibial surface tends to internal rotation. In the same paper, Cobb et al. [34] compared the newly determined ATA to perpendicular to the posterior tibial condylar axis (PTCA) and found that latter is 6 degrees internally rotated. The PTCA has been found the most consistent landmark at different levels of resection of the tibia and the authors suggest 10 degrees of external rotation to the line perpendicular to it as the ideal component rotation [26]. How the authors elaborated the 10 degrees of external rotation is not described; however, if we accept that, this means that Cobb et al.'s proposed axis (ATA) - which is based on condylar contours and derived condylar centres - would be 4 degrees in internal rotation.

Thus our study confirms these calculations.

Rotating platform designs can theoretically compensate for malrotation of the tibial component, on the other hand, they may lead to increased rotational instability [37]. External rotation laxity has been shown to be predominant in these cases.

Zhao et al. [38] examined the external rotation of the femoral component in the setting of dynamic implant orientation and found a mean 5.7 degrees of external rotation to be necessary in contrast to the conventionally applied three degrees. The effects on tibial component rotational alignment have not been examined, but these findings suggest that an increased external rotation on the tibial implant may be beneficial. In another study, less than 2 degrees of external rotation of the tibial component was found to be a risk factor for reduced component survival in TKA [39].

Reproducing exact degrees of component rotation appears to be difficult. New systems, such as portable navigation systems, aim to minimise invasiveness and amount of material used in older navigation systems [40]. However, little attention has been given to tibial rotation when navigation was applied. The pinless navigation described by Maderbacher at al. [41], taking the Akagi-line [29] as a reference, may represent a good approach, but we think that further comparative anatomical studies are required to correlate it with the PTCA.

In order to compensate for the internal rotation shift with tibial resection, as has been shown in this study, we propose referencing the posterior condylar axis and then adding 10 degrees of external rotation, as the latter is a constant landmark independent of resection depth [26]. In this case, however, a posteriorly uncovered area of the medial tibial condyle should be seen with symmetric implants.

The difficulty of how to avoid posterolateral overhang and internal rotation, and yet gain maximum coverage with a symmetrical tibial component, led to the development of asymmetric implants [42].

Furthermore, we suggest that designs be produced with a more posterior lateral contact area representing the physiological articular surface centre in order to avoid internal rotation errors.

Conflict of interest The authors declare that they have no conflict of interest.

\section{References}

1. Ethgen O, Bruyere O, Richy F, Dardennes C, Reginster JY (2004) Health-related quality of life in total hip and total knee arthroplasty. A qualitative and systematic review of the literature. J Bone Joint Surg Am 86-A(5):963-974

2. van Essen GJ, Chipchase LS, O'Connor D, Krishnan J (1998) Primary total knee replacement: short-term outcomes in an Australian population. J Qual Clin Pract 18(2):135-142

3. Jacobs CA, Christensen CP, Karthikeyan T (2014) Patient and intraoperative factors influencing satisfaction two to five years after primary total knee arthroplasty. J Arthroplasty 29(8):1576-1579. doi:10.1016/j.arth.2014.03.022

4. Sesen H, Demirkale I, Karaduman M, Vural CA, Okkaoglu MC, Altay M (2014) Why two-thirds of patients accepted the second session in staged bilateral total knee arthroplasty: a prospective analysis of 111 patients. Knee Surg Sports Traumatol Arthrosc. doi:10.1007/s00167-014-3251-7

5. Bellemans J, Robijns F, Duerinckx J, Banks S, Vandenneucker H (2005) The influence of tibial slope on maximal flexion after total knee arthroplasty. Knee Surg Sports Traumatol Arthrosc 13(3): 193-196. doi:10.1007/s00167-004-0557-x 
6. Briard JL, Witoolkollachit P, Lin G (2007) Soft tissue management in total knee replacement. Analysis of ligament balancing. Orthopade 36(7):635-642. doi:10.1007/s00132-007-1109-0

7. Kurtz S, Ong K, Lau E, Mowat F, Halpern M (2007) Projections of primary and revision hip and knee arthroplasty in the United States from 2005 to 2030. J Bone Joint Surg Am 89(4):780-785. doi:10. 2106/JBJS.F.00222

8. Dalury DF, Pomeroy DL, Gorab RS, Adams MJ (2013) Why are total knee arthroplasties being revised? J Arthroplasty 28(8 Suppl): 120-121. doi:10.1016/j.arth.2013.04.051

9. Sharkey PF, Hozack WJ, Rothman RH, Shastri S, Jacoby SM (2002) Insall Award paper. Why are total knee arthroplasties failing today? Clin Orthop Relat Res 404:7-13

10. Hutter EE, Granger JF, Beal MD, Siston RA (2013) Is there a gold standard for TKA tibial component rotational alignment? Clin Orthop Relat Res 471(5):1646-1653. doi:10.1007/s11999-0132822-0

11. Petersen W, Rembitzki IV, Bruggemann GP, Ellermann A, Best R, Koppenburg AG, Liebau C (2014) Anterior knee pain after total knee arthroplasty: a narrative review. Int Orthop 38(2):319-328. doi:10.1007/s00264-013-2081-4

12. Bedard M, Vince KG, Redfern J, Collen SR (2011) Internal rotation of the tibial component is frequent in stiff total knee arthroplasty. Clin Orthop Relat Res 469(8):2346-2355. doi:10.1007/s11999011-1889-8

13. Barrack RL, Schrader T, Bertot AJ, Wolfe MW, Myers L (2001) Component rotation and anterior knee pain after total knee arthroplasty. Clin Orthop Relat Res 392:46-55

14. Lakstein D, Zarrabian M, Kosashvili Y, Safir O, Gross AE, Backstein D (2010) Revision total knee arthroplasty for component malrotation is highly beneficial: a case control study. J Arthroplasty 25(7):1047-1052. doi:10.1016/j.arth.2009.07.004

15. Luyckx T, Peeters T, Vandenneucker H, Victor J, Bellemans J (2012) Is adapted measured resection superior to gap-balancing in determining femoral component rotation in total knee replacement? J Bone Joint Surg (Br) 94(9):1271-1276. doi:10.1302/0301-620X. 94B9.28670

16. Fehring TK (2000) Rotational malalignment of the femoral component in total knee arthroplasty. Clin Orthop Relat Res 380:72-79

17. Kinzel V, Ledger M, Shakespeare D (2005) Can the epicondylar axis be defined accurately in total knee arthroplasty? Knee 12(4): 293-296. doi:10.1016/j.knee.2004.09.003

18. Talbot S, Dimitriou P, Radic R, Zordan R, Bartlett J (2014) The sulcus line of the trochlear groove is more accurate than Whiteside's Line in determining femoral component rotation. Knee Surg Sports Traumatol Arthrosc. doi:10.1007/s00167-014-3137-8

19. Kobayashi H, Akamatsu Y, Kumagai K, Kusayama Y, Ishigatsubo R, Muramatsu S, Saito T (2014) The surgical epicondylar axis is a consistent reference of the distal femur in the coronal and axial planes. Knee Surg Sports Traumatol Arthrosc. doi:10.1007/ s00167-014-2867-y

20. Incavo SJ, Coughlin KM, Pappas C, Beynnon BD (2003) Anatomic rotational relationships of the proximal tibia, distal femur, and patella: implications for rotational alignment in total knee arthroplasty. J Arthroplasty 18(5):643-648

21. Brooks P (2009) Seven cuts to the perfect total knee. Orthopedics 32 (9). doi:10.3928/01477447-20090728-27

22. Dalury DF (2001) Observations of the proximal tibia in total knee arthroplasty. Clin Orthop Relat Res 389:150-155

23. Siston RA, Goodman SB, Patel JJ, Delp SL, Giori NJ (2006) The high variability of tibial rotational alignment in total knee arthroplasty. Clin Orthop Relat Res 452:65-69. doi:10.1097/01. blo.0000229335.36900.a0

24. Eckhoff DG, Metzger RG, Vandewalle MV (1995) Malrotation associated with implant alignment technique in total knee arthroplasty. Clin Orthop Relat Res 321:28-31
25. Huddleston JI, Scott RD, Wimberley DW (2005) Determination of neutral tibial rotational alignment in rotating platform TKA. Clin Orthop Relat Res 440:101-106

26. Graw BP, Harris AH, Tripuraneni KR, Giori NJ (2010) Rotational references for total knee arthroplasty tibial components change with level of resection. Clin Orthop Relat Res 468(10):2734-2738. doi: 10.1007/s11999-010-1330-8

27. Ikeuchi M, Yamanaka N, Okanoue Y, Ueta E, Tani T (2007) Determining the rotational alignment of the tibial component at total knee replacement: a comparison of two techniques. J Bone Joint Surg (Br) 89(1):45-49. doi:10.1302/0301-620X.89B1. 17728

28. Akagi M, Mori S, Nishimura S, Nishimura A, Asano T, Hamanishi C (2005) Variability of extraarticular tibial rotation references for total knee arthroplasty. Clin Orthop Relat Res 436:172-176

29. Akagi M, Oh M, Nonaka T, Tsujimoto H, Asano T, Hamanishi C (2004) An anteroposterior axis of the tibia for total knee arthroplasty. Clin Orthop Relat Res 420:213-219

30. Kaech AMN, Müller-Gerbl M (2002) Implementierung eines standardisierten Koordinatensystems für dreidimensionale CTDarstellungen des Kniegelenks mit VGStudio max. Dissertation, University of Basel, Basel, pp 1-33

31. Nowakowski AM, Muller-Gerbl M, Valderrabano V (2012) Assessment of knee implant alignment using coordinate measurement on three-dimensional computed tomography reconstructions. Surg Innov 19(4):375-384. doi:10.1177/1553350611429689

32. Grood ES, Suntay WJ (1983) A joint coordinate system for the clinical description of three-dimensional motions: application to the knee. J Biomech Eng 105(2):136-144

33. McPherson A, Karrholm J, Pinskerova V, Sosna A, Martelli S (2005) Imaging knee position using MRI, RSA/CT and 3D digitisation. J Biomech 38(2):263-268. doi:10.1016/j.jbiomech. 2004.02.007

34. Cobb JP, Dixon H, Dandachli W, Iranpour F (2008) The anatomical tibial axis: reliable rotational orientation in knee replacement. J Bone Joint Surg (Br) 90(8):1032-1038. doi:10.1302/0301-620X. 90B8.19905

35. Cheng FB, Ji XF, Lai Y, Feng JC, Zheng WX, Sun YF, Fu YW, Li YQ (2009) Three dimensional morphometry of the knee to design the total knee arthroplasty for Chinese population. Knee 16(5):341347. doi:10.1016/j.knee.2008.12.019

36. Baier C, Fitz W, Craiovan B, Keshmiri A, Winkler S, Springorum R, Grifka J, Beckmann J (2014) Improved kinematics of total knee replacement following partially navigated modified gap-balancing technique. Int Orthop 38(2):243-249. doi:10.1007/s00264-0132140-x

37. Mayr HO, Reinhold M, Hube R, von Roth P, Bernstein A, Suedkamp N, Stoehr A (2014) Rotational laxity and collateral ligament laxity following total knee arthroplasty with rotating platform. Int Orthop 38(7):1379-1386. doi:10.1007/ s00264-014-2308-Z

38. Zhao Z, Wang W, Wang S, Jiang L, Zhang S, Zhao Y (2015) Femoral rotation influences dynamic alignment of the lower extremity in total knee arthroplasty. Int Orthop 39(1):55-60. doi:10. 1007/s00264-014-2484-x

39. Kim YH, Park JW, Kim JS, Park SD (2014) The relationship between the survival of total knee arthroplasty and postoperative coronal, sagittal and rotational alignment of knee prosthesis. Int Orthop 38(2):379-385. doi:10.1007/s00264-013-2097-9

40. Iorio R, Mazza D, Drogo P, Bolle G, Conteduca F, Redler A, Valeo L, Conteduca J, Ferretti A (2015) Clinical and radiographic outcomes of an accelerometer-based system for the tibial resection in total knee arthroplasty. Int Orthop 39(3):461-466. doi:10.1007/ s00264-014-2541-5 
41. Maderbacher G, Schaumburger J, Keshmiri A, Barthel M, Springorum HR, Craiovan B, Grifka J, Baier C (2015) Pinless navigation in total knee arthroplasty: Navigation reduced by the maximum? Int Orthop 39(3):455-460. doi:10.1007/s00264-014-2529-1
42. Crockarell JR Jr, Hicks JM, Schroeder RJ, Guyton JL, Harkess JW, Lavelle DG (2010) Total knee arthroplasty with asymmetric femoral condyles and tibial tray. J Arthroplasty 25(1):108-113. doi:10. 1016/j.arth.2008.11.002 